\title{
Effects of Semen Processing on Sperm Function: Differences between Swim-Up and Density Gradient Centrifugation
}

\author{
Gabriela Hernández-Silva ${ }^{1} *$ (iD) , Aideé S. López-Torres ${ }^{1} *$ (iD), Israel Maldonado-Rosas ${ }^{2}$ (iD, \\ Esperanza Mata-Martínez ${ }^{3, * *(\mathbb{D})}$, Fernando Larrea ${ }^{10}$, Víctor Torres-Flores ${ }^{4}$ (i) , Claudia L. Treviño $^{3}$, \\ Mayel Chirinos ${ }^{1} \mathbb{D}$ \\ ${ }^{1}$ Department of Reproductive Biology Dr. Carlos Gual Castro, Instituto Nacional de Ciencias Médicas y Nutrición Salvador Zubirán, ${ }^{2}$ Centro \\ de Innovación Tecnológica y Medicina Reproductiva (Citmer), Mexico City, ${ }^{3}$ Department of Developmental Genetics and Molecular \\ Physiology, Instituto de Biotecnología, UNAM, Cuernavaca, ${ }^{4}$ Laboratory of Biomembranes, Faculty of Medicine, Universidad Nacional \\ Autónoma de México, Mexico City, Mexico
}

Purpose: Andrology research has evolved notoriously in the latest years, particularly since male factor contribution to couple infertility has been undoubtedly demonstrated. However, sperm function investigations results are sometimes contradictory, probably as a result of the use of different sperm processing techniques. In this work, we underwent a systematic functional comparison of human sperm samples simultaneously processed by swim-up and density gradient centrifugation, which are the preferred sperm processing methods used in basic and clinical laboratories.

Materials and Methods: To compare functional characteristics of sperm isolated by swim-up and density gradient centrifugation followed by incubation at different times under capacitating conditions.

Results: Semen samples processed in parallel by these two procedures resulted in sperm preparations with significant differences in redox state, spontaneous intracellular calcium oscillations, hyperactivation, protein tyrosine phosphorylation, and acrosome reaction responsivity to calcium ionophore. Such differences showed time-dependent specific patterns for spontaneous intracellular calcium oscillations, hyperactivation and protein tyrosine phosphorylation. Sperm retrieved by density gradient centrifugation showed more hyperactivation and tyrosine phosphorylation than swim-up sperm, suggesting a higher degree of capacitation.

Conclusions: Our results account for functional differences observed in spermatozoa processed with these two methods and therefore may contribute to a better interpretation of outcomes obtained in different laboratories as well as to improve experimental designs aimed to study sperm physiology and fertility potential.

Keywords: Fertility; Sperm capacitation; Sperm retrieval; Spermatozoa

This is an Open Access article distributed under the terms of the Creative Commons Attribution Non-Commercial License (http://creativecommons.org/licenses/by-nc/4.0) which permits unrestricted non-commercial use, distribution, and reproduction in any medium, provided the original work is properly cited.

Received: Jun 26, 2020 Revised: Aug 21, 2020 Accepted: Oct 21, 2020 Published online Nov 19, 2020

Correspondence to: Mayel Chirinos (iD https://orcid.org/0000-0003-0023-5195

Department of Reproductive Biology Dr. Carlos Gual Castro, Instituto Nacional de Ciencias Médicas y Nutrición Salvador Zubirán, Vasco de Quiroga 15, Tlalpan 14080, México City, México.

Tel: +52-55-54870900 (ext. 2417), E-mail: mayel.chirinose@incmnsz.mx

*These authors contributed equally to this work as co-first authors.

${ }^{* *}$ Current address: Instituto de Histología y Embriología de Méndoza (IHEM) Dr. Mario H. Burgos, CONICET, Universidad Nacional de Cuyo, Mendoza, Argentina. 


\section{INTRODUCTION}

There are several standardized semen processing protocols for sperm retrieval in andrology laboratories and the most widely employed are the direct swim-up (SU) and the discontinuous density gradient centrifugation (DGC), both documented and recommended by the World Health Organization (WHO) manual for obtaining motile spermatozoa enriched with morphologically normal forms and free of seminal plasma, debris, non-germ cells and dead spermatozoa [1]. Studies comparing these two sperm preparation techniques have focused in investigating recovery rates and conventional sperm parameters, such as motility and morphology [2,3], resulting in general recommendations for their preferred use for different assisted reproduction techniques (ART). Besides rendering sperm preparations with higher total and progressive motility than the unprocessed samples, clinical data including results of efficacy in ART outcomes seem to support that SU and DGC also bring comparable spermatozoa in terms of morphology [4], hyaluronan binding capacity [5], telomeres length [6], and apoptosis [7]. Moreover, they are also effective in eliminating spermatozoa with DNA damage [8] and selecting spermatozoa with longer telomeres [6].

Sperm capacitation comprises a set of sequential changes taking place during female tract residency that enables this cell to fertilize. Some of the best characterized changes associated to sperm capacitation are cholesterol efflux from plasma membrane, intracellular calcium increase, motility pattern changes (including the appearance of hyperactivated motility), increase in proteins tyrosine phosphorylation and the ability to undergo the acrosome reaction [9]. These changes initiates after leaving the seminal plasma and are subsequently modulated by sperm interaction with molecules from the female reproductive tract, such as reproductive hormones [10] and secreted proteins [11]. Sperm processing techniques are intended to separate viable cells from seminal plasma, and therefore stimulate capacitation. Although SU and DGC have been indistinctively employed for sperm recovery from semen, some evidences indicate quality and functional differences between cells obtained by these two methods. For instance, SU preparations have shown a greater incidence of cells with high levels of intracellular reactive oxygen species (ROS) and lower mitochondrial membrane potential compared to DGC [12]. Besides, a recent investigation showed that DNA fragmentation is higher in DGC-processed spermatozoa than in those recovered by SU [13].

Even though molecules and mechanisms regulating the progress of human sperm capacitation have been intensively investigated, the consequences of preparation techniques on sperm function under capacitating conditions have not been examined. Therefore, the aim of this investigation was to compare the effects of SU and DGC on several sperm function variables associated with capacitation.

\section{MATERIALS AND METHODS}

\section{Ethics statement}

This study was approved by the Ethics and Research Committees of the Instituto Nacional de Ciencias Médicas y Nutrición Salvador Zubirán (Reg. No. 2600) and all volunteers signed an informed written consent form.

\section{Semen samples and sperm preparation}

Healthy normozoospermic donors $(n=16)$ provided fresh ejaculates by masturbation after $3-5$ days of sexual abstinence. Semen samples were allowed to liquefy for at least 30 minutes at $37^{\circ} \mathrm{C}$ and assessed following WHO standard procedures and reference values [1]. Each semen sample was split in two fractions for simultaneous sperm isolation by DGC and SU, following procedures previously described. For preparation of DGC-sperm samples, semen was centrifuged at $800 \times g$ for 30 minutes through $90 / 50 \%$ discontinuous density gradients (Isolate; Irvine Scientific, Irvine, CA, USA). Sperm pellets were washed with human tubal fluid (HTF) medium ( $\mathrm{pH}$ 7.4) by centrifugation at $800 \times g$ for 10 minutes and resuspended in HTF supplemented with $0.3 \%$ human serum albumin and $0.3 \mathrm{mM}$ sodium pyruvate (supplemented HTF, sHTF) [14]. For obtaining SU-sperm samples, $200 \mu \mathrm{L}$ aliquots of semen were overloaded with $800 \mu \mathrm{L}$ of sHTF and incubated for 1 hour at $37^{\circ} \mathrm{C}$ in an atmosphere of $5 \% \mathrm{CO}_{2}$ in air with the tubes inclined at an angle of $45^{\circ}$. Subsequently, the uppermost $750 \mu \mathrm{L}$ with motile cells were carefully collected [15]. Sperm preparations obtained after both procedures were re-assessed for sperm count and motility and cell concentrations were adjusted to $15 \times 10^{6}$ sperm/ $\mathrm{mL}$ in sHTF. Spermatozoa obtained under these condi- 
tions were considered as SU- or DGC-sperm at time zero and further incubations at longer times were performed in sHTF under capacitating conditions $\left(37^{\circ} \mathrm{C}, 5 \%\right.$ $\mathrm{CO}_{2}$ in air). Only sperm samples with a total motility above $80 \%$ after processing were used for experiments. A flow diagram depicting the experimental design is presented in Supplement Fig. 1.

\section{Evaluation of redox potential}

The oxidation-reduction potential (ORP) is a measure of redox imbalance and was evaluated using the MiOXSYS system (Aytu Bioscience, Englewood, CO, USA) [16]. For static ORP (sORP) quantification, $30 \mu \mathrm{L}$ of sperm were loaded on the sample port of disposable sensors and then inserted into the sensor module of the MiOXSYS analyzer. The analysis started automatically when the sample reached the reference cell and the electrochemical circuit was completed. The sORP values displayed in millivolts $(\mathrm{mV})$ were expressed as $\mathrm{mV} / 10^{6}$ sperm $/ \mathrm{mL}$.

\section{Spontaneous intracellular calcium oscillations in sperm}

Analysis of intracellular calcium changes were performed in single cells as described before [14,17]. SUand DGC-sperm were loaded with $2 \mu \mathrm{M}$ Fluo-3 AM (Invitrogen, Eugene, OR, USA) in HTF devoid of albumin and further incubated for 30 minutes at $37^{\circ} \mathrm{C}$. After washing, spermatozoa were attached by the heads to $0.03 \%$ poly-L-lysine pre-treated coverslips and mounted in a recording chamber (Harvard Apparatus, Holliston, MA, USA) standing on the thermo-regulated platform of an inverted fluorescence microscope (Olympus IX71; Olympus, Tokyo, Japan). Videos were acquired using the $60 \times$ microscope objective coupled to a camera (iXon 888; Andor Bioimaging, Wilmington, NC, USA) with the IQ software (Andor Bioimaging). Fluorescence images were collected every 500 mseconds and recorded for 360 seconds before $3 \mu \mathrm{M}$ progesterone $(\mathrm{Pg})$ was added as positive control. Raw fluorescence intensity values were retrieved from videos using the ImageJ free software and fluorescence intensity (\%) was calculated by normalizing fluorescence data $(\mathrm{F})$ with respect to the maximum intensity obtained after Pg addition (considered as 100\%) for each cell, after subtraction of the minimum intensity value observed during the first minute, using the equation: $\left(\mathrm{F}_{\mathrm{X}}-\mathrm{F}_{\mathrm{Min}}\right) \times 100 /\left(\mathrm{F}_{\mathrm{Pg}}-\mathrm{F}_{\mathrm{Min}}\right)$. The total recorded series were plotted against time and only oscillations with fluorescence intensity above $10 \%$ were considered for analysis. Oscillations frequency (number of oscillations/min) and amplitude (average of maximum fluorescence intensities from each trace oscillations) were assessed.

\section{Motility evaluated by Computer-Aided Sperm Analyzer}

SU- or DGC-sperm samples were placed in $20 \mu \mathrm{m}$ deep cell counting chambers (Leja Products BV, GN NieuwVennep, Netherlands) and evaluated by a ComputerAided Sperm Analyzer (CASA; Hamilton Thorne IVOS version 14.0; Hamilton Thorne Research Inc., Beverly, MA, USA), operating at a rate of $30 \mathrm{frames} / \mathrm{s}$ and a frequency of $60 \mathrm{~Hz}$. Seven to ten random fields were evaluated for a minimum of 300 cells per experimental condition. Percentage of total motile cells and kinetic variables values were retrieved and percentages of hyperactivated cells were estimated using curvilinear velocity (VCL) $\geq 150$, linearity (LIN) $\leq 50$ and amplitude of lateral head displacement $(\mathrm{ALH}) \geq 3.5$ as cut-off values [15].

\section{Sperm proteins tyrosine phosphorylation}

Protein tyrosine phosphorylation was evaluated by Western blot. Sperm aliquots equivalent to $1.2 \times 10^{6}$ cells were washed twice with phosphate buffer saline (PBS) supplemented with sodium orthovanadate $(1 \mathrm{mM})$ and genistein $(0.02 \mathrm{mM})$. Afterwards, sperm pellets were resuspended in denaturing buffer ( $2 \%$ sodium dodecyl sulphate [SDS], $10 \%$ glycerol, $2 \% \beta$-mercaptoethanol, $0.61 \mathrm{M}$ Tris- $\mathrm{HCl} \mathrm{pH}$ 6.8) and boiled for 10 minutes for SDS-polyacrylamide gel electrophoresis. After electrophoresing, gels were electrotransferred onto nitrocellulose membranes and further blocked with bovine serum albumin $3 \%$ in tris-buffered saline (TBS)-Tween $20(0.05 \%)$. Immunodetection was performed by probing membranes with a monoclonal antibody against phosphotyrosine residues (anti-pY, 1:5,000, clone 4G10; Millipore, Temeluca, CA, USA). After three washes with TBS-Tween 20, membranes were incubated in the presence of horseradish peroxidase-conjugated antimouse immunoglobulin G (1:4,000; Millipore) and immunocomplexes were detected by enhanced chemiluminescence on a Chemidoc XRS+ imaging system (Bio-Rad, Hercules, CA, USA). Homogeneous protein loading was corroborated after membrane stripping for re-probing with a mouse monoclonal anti- $\beta$-tubulin (anti-tub; 
1:50,000; Sigma-Aldrich, St. Louis, MO, USA). Densitometric analyses were performed using the ImageLab software (version 4; Bio-Rad). Results were expressed as the relative density of phosphotyrosine/ $\beta$-tubulin $(\mathrm{pY} /$ tub).

\section{Acrosome reaction assessment}

Spontaneous and calcium ionophore-induced acrosome reaction were assessed after staining with fluorescein isothiocyanate-conjugated Pisum sativum agglutinin (FITC-PSA) as previously described [18] Briefly, two sperm aliquots of 150,000 cells from each experimental condition were used for immediate fixation with $70 \%$ ethanol or fixation after incubation with the calcium ionophore A23187 $(10 \mu \mathrm{M})$ (Sigma-Aldrich) during 20 minutes at $37^{\circ} \mathrm{C}$. Subsequently, sperm samples were smeared onto poly-L-lysine pretreated slides and stained with $20 \mu \mathrm{g} / \mathrm{mL}$ of FITC-PSA (SigmaAldrich) for 30 minutes. After two washes of 5 minutes with PBS, at least two hundred cells were evaluated under a fluorescence microscope to assess the acrosomal status and determine the percentage of acrosomereacted cells. Data were expressed as the acrosome reaction to ionophore challenge (ARIC) score, defined as the percentage of calcium ionophore-induced acrosome reaction minus the percentage of spontaneous acrosome reaction [15].

\section{Statistical analysis}

Results were expressed as the mean \pm standard error of mean of a minimum of three independent experiments. Comparisons between SU- and DGC-sperm data were performed using the GraphPad Prism software (version 5.01; GraphPad Software, San Diego, CA, USA). Data from all analyzed variables were subjected to the Wilcoxon matched-pairs signed-ranks test to evaluate differences between the two groups, except for sperm intracellular calcium oscillations changes that were analyzed using the unpaired t-test. Values were considered significantly different at $\mathrm{p}<0.05$.

\section{RESULTS}

Semen samples were subjected to an initial analysis and all were classified as normozoospermic. No sample required to be excluded. Fresh semen as well as SUand DGC-sperm characteristics are presented in Table 1.

\section{Density gradient centrifugation-sperm have higher redox potential than swim-up- sperm}

The initial approach to evaluate potential differences between SU- and DGC-sperm was to assess their redox potential at time zero. As shown in Table 1, DGCsperm exhibited significant higher sORP values than SU-sperm. Additionally, redox potential of SU- and DGC-sperm at 2, 4, and 6 hours incubations was also evaluated but did not exhibit significant differences compared to time zero (data not shown).

\section{Swim-up- and density gradient centrifugation-sperm show different intracellular calcium oscillations patterns}

As calcium influx is one of the earliest changes during sperm capacitation, we evaluated the effects of SU and DGC on intracellular calcium dynamics in single cells. Representative spermatozoa intracellular calcium traces from each treatment at time zero and 4 hours incubations are shown (Fig. 1A). When comparing the percentage of cells showing intracellular calcium oscillations in each condition, we found DGC-sperm showed significantly higher incidence of calcium oscillating

Table 1. Sperm parameters and redox state of normozoospermic semen samples before and after processing by SU and DGC (time zero) ( $n=16$ )

\begin{tabular}{lccc}
\multicolumn{1}{c}{ Parameter } & Fresh semen sample & SU & DGC \\
\hline Age $(\mathrm{y})$ & $26.75 \pm 1.07$ & - & - \\
Volume $(\mathrm{mL})$ & $3.38 \pm 0.31$ & $1.69 \pm 0.15$ & $1.03 \pm 0.14$ \\
Density $\left(\times 10^{6}\right.$ sperm $\left./ \mathrm{mL}\right)$ & $129.18 \pm 23.30$ & $43.29 \pm 8.56$ & $91.40 \pm 13.63$ \\
Total sperm count $\left(\times 10^{6}\right)$ & $349.41 \pm 82.15$ & $59.02 \pm 17.83$ & $77.03 \pm 18.84$ \\
Motility $(\%)$ & $79.81 \pm 2.63$ & $86.25 \pm 1.64$ & $81.44 \pm 1.25^{*}$ \\
SORP $\left(\mathrm{mV} / 10^{6}\right.$ sperm $\left./ \mathrm{mL}\right)$ & $0.74 \pm 0.13$ & $7.81 \pm 0.38$ & $16.62 \pm 0.80^{*}$ \\
\hline
\end{tabular}

Values are presented as mean \pm standard error of mean.

SU: swim-up, DGC: density gradient centrifugation, sORP: static oxidation-reduction potential.

${ }^{*} \mathrm{p}<0.05$ vs. SU. 
cells than SU-sperm at time zero but this difference disappeared after 4 hours incubations (Fig. 1B). Likewise, DGC-sperm displayed intracellular calcium oscillations with higher frequency than SU-sperm at time zero and 4 hours (Fig. 1C). Oscillations amplitude was also significantly higher in DGC-sperm than in SU- sperm but only at time zero (Fig. 1D). In contrast, DGCsperm showed non-significant differences between time zero and 4 hours incubations for percentage of cells showing calcium oscillations, frequency and amplitude, while SU-sperm exhibited an increase in the percentage of cells showing calcium oscillations and frequency
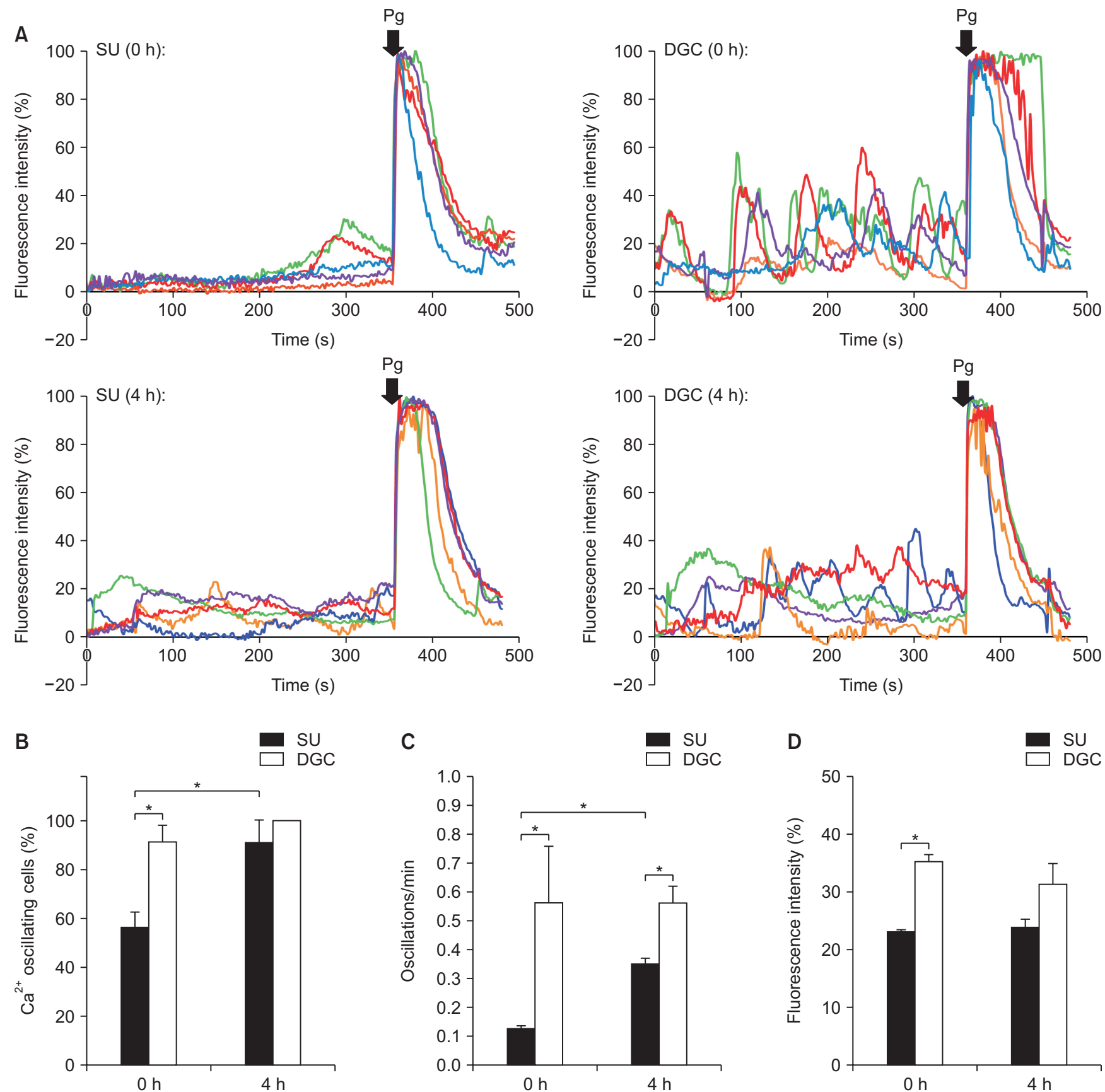

C
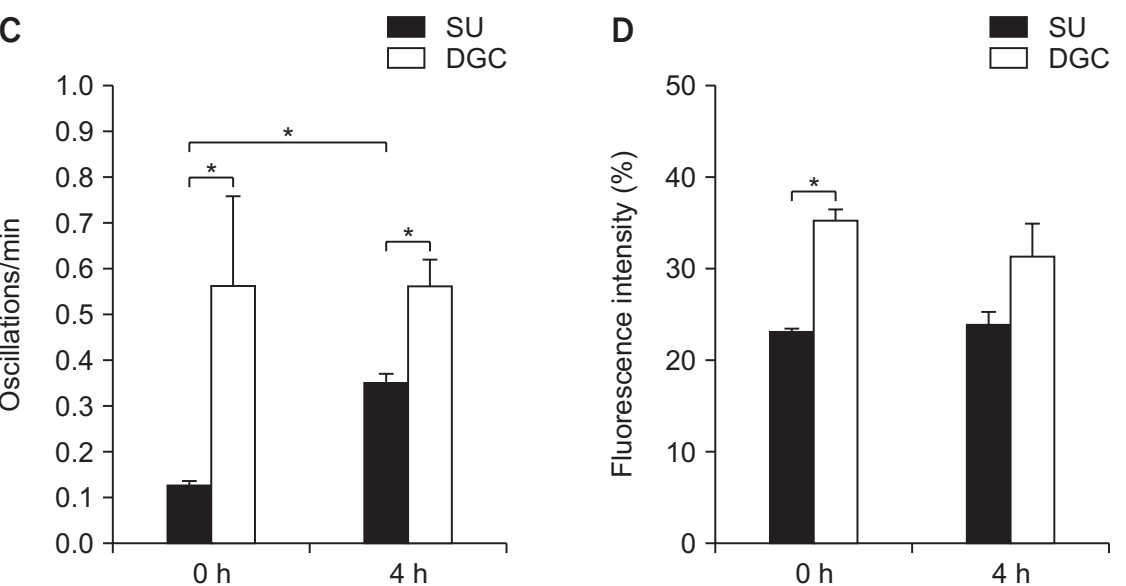

Fig. 1. Intracellular calcium oscillations of spermatozoa after swim-up (SU) and density gradient centrifugation (DGC) ( $n=3)$. (A) Representative calcium traces from 5 spermatozoa obtained by SU and DGC at 0 and 4 hours incubations under capacitating conditions. (B) Percentage of cells exhibiting intracellular calcium oscillations after SU and DGC preparation at 0 and 4 hours incubations under capacitating conditions. (C) Frequency (oscillations/min) and (D) amplitude (fluorescence intensity) of SU- and DGC-sperm intracellular calcium oscillations at 0 and 4 hours incubations under capacitating conditions. Pg: progesterone. ${ }^{*} \mathrm{p}<0.05$. Total cells analyzed at 0 hour incubations were 51 for SU and 40 for DGC; and at 4 hours incubations were 36 for SU and 43 for DGC. 
after capacitation for 4 hours.

\section{Density gradient centrifugation-sperm} hyperactivate faster than swim-up-sperm

A time-course analysis of motility on SU- and DGCsperm showed no significant differences in the kinetic parameters analyzed by CASA, except for VCL at 4 and 6 hours incubations under capacitating conditions (Supplement Table 1). When the incidence of hyperactivated cells was assessed, SU- and DGC-sperm showed similar values at time zero, followed by a time dependent increase of hyperactivation in both sperm preparations. However, the percentage of hyperactivation in DGC-sperm was higher than in SU-sperm at 2, 4, and 6 hours incubations (Fig. 2A), regardless the percentages of total motility that showed no significant differences at any incubation time (Fig. 2B).

\section{Density gradient centrifugation- sperm exhibit higher protein tyrosine phosphorylation than swim-up-sperm at short incubation times}

When the effects of SU and DGC on sperm tyrosine phosphorylation were evaluated, we found a timedependent upsurge of the phosphotyrosine signal intensity with both processing. However, DGC-sperm showed significantly higher levels of phosphotyrosine signal than their SU-sperm counterparts at all incubation times except at 6 hours (Fig. 3).

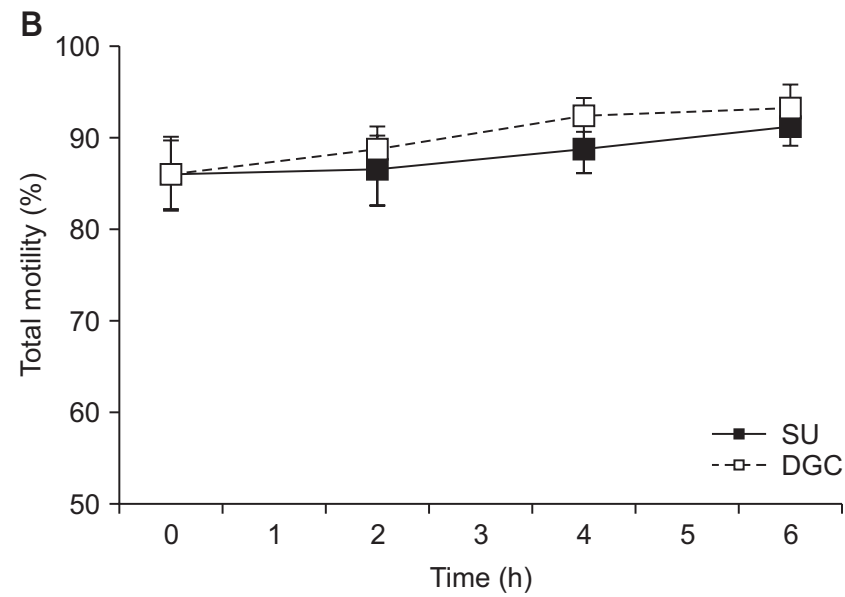

Fig. 2. Effects of swim-up (SU) and density gradient centrifugation (DGC) on hyperactivation ( $n=7)$. (A) Percentage of hyperactivated cells. (B) Percentage of total sperm motility in same sample sets. ${ }^{*} \mathrm{p}<0.05 \mathrm{vs}$. SU at same incubation time.

A

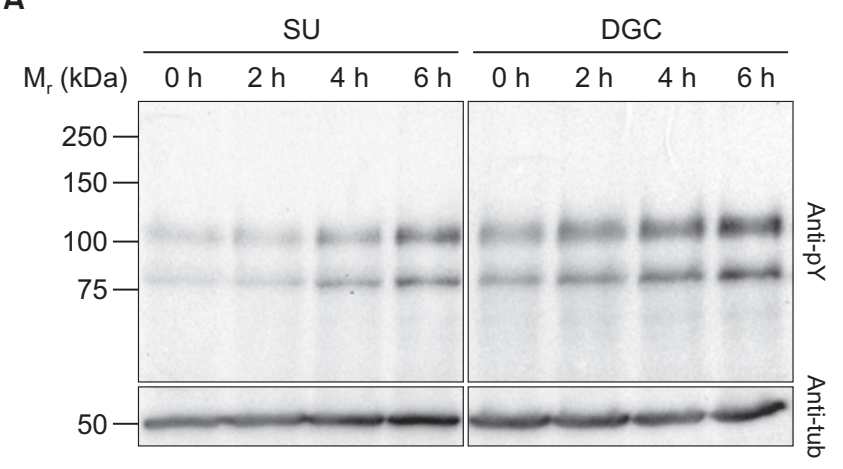

B

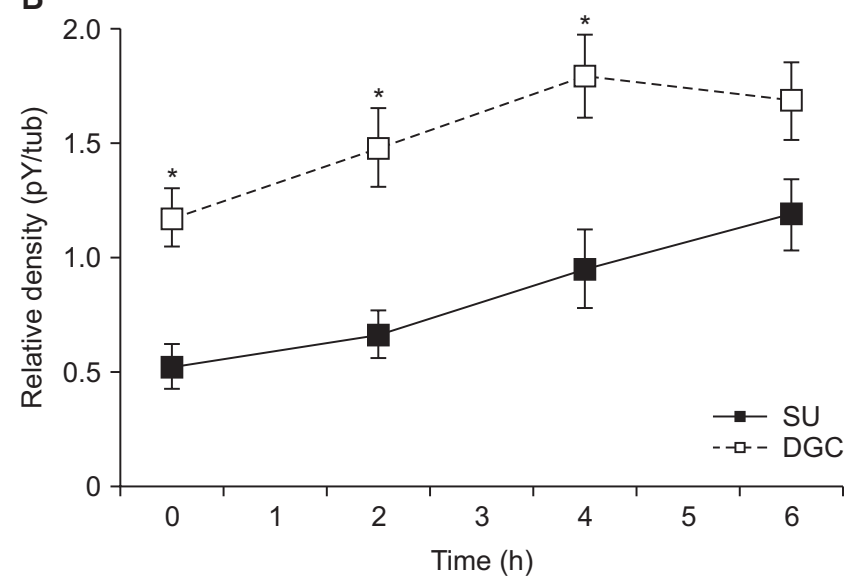

Fig. 3. Protein tyrosine phosphorylation changes in spermatozoa obtained by swim-up (SU) and density gradient centrifugation (DGC) ( $\mathrm{n}=7$ ). Changes in SU- and DGC-sperm protein tyrosine phosphorylation (pY) were evaluated by Western blot. After pY detection, membranes were reprobed for $\beta$-tubulin (tub) to be used as loading control. (A) Representative Western blots of $\mathrm{pY}$ and tub. (B) Densitometric analysis of normalized intensities (pY/tub). ${ }^{*} p<0.05$ vs. SU at same incubation time. 


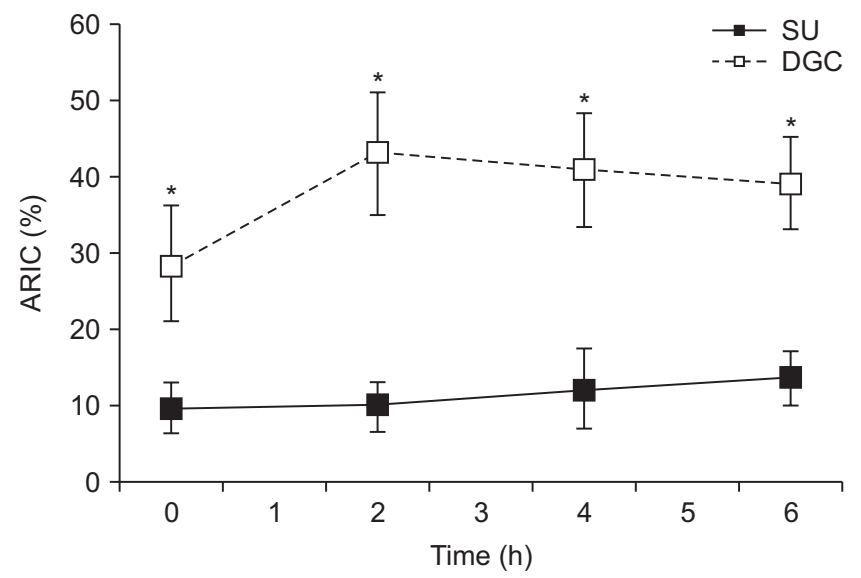

Fig. 4. Acrosome reaction to ionophore challenge (ARIC) in spermatozoa obtained by swim-up (SU) and density gradient centrifugation (DGC) $(n=8)$. Spermatozoa were capacitated at different times and challenged with calcium ionophore A23187. After evaluation of the percentage of acrosome-reacted cells in control and calcium ionophore-challenged paired-aliquots, the ARIC were estimated. ${ }^{*} \mathrm{p}<0.05$ vs. SU at same incubation time.

\section{Density gradient centrifugation stimulates sperm acrosome reaction responsivity to calcium ionophore}

To further evaluate the effects of sperm processing on sperm, spontaneous and calcium ionophore-induced acrosome reaction was assessed and the ARIC index was estimated. The results showed that DGC-sperm had a significantly higher ability to undergo calcium ionophore-induced acrosome reaction than $\mathrm{SU}$-sperm at time zero and 2, 4, and 6 hours incubations (Fig. 4).

\section{DISCUSSION}

In vivo, spermatozoa must leave the seminal fluid in order to capacitate and fertilize and consequently several methods of sperm recovery from semen have been implemented for ART. SU and DGC are broadly used for sperm processing and have been indistinctly used in basic and clinical andrology laboratories for many years, but potential differences between them may have led to erroneous interpretation of results on sperm capacitation and fertilization studies. Indeed, although both methods have proved to render high quality spermatozoa with comparable characteristics regarding motility, morphology and sperm count [12], several investigations suggest molecular and functional differences between SU- and DGC-sperm. For example, prostatic zinc levels in sperm obtained by SU are significantly higher than in DGC preparations, suggesting different levels of seminal plasma contamination [19], which is relevant for selection of the sperm processing method since seminal plasma contaminants may act as decapacitating agents [15]. Furthermore, a proteomic comparison between DGC and SU processed sperm indicates possible dissimilarities in their glycolytic metabolism and DNA methylation and suggests DGC cells may have a better capacitation potential [20]. Consequently, sperm processing is likely to have effects on sperm function, capacitation and fertility.

In the present study, sperm selected after SU and DGC showed significant differences regarding several functional and capacitation related characteristics of the sperm. Physiological levels of ROS are necessary for optimal sperm functions such as motility, hyperactivation, capacitation, acrosome reaction, and sperm capabilities to fertilize [21], but oxidative stress occurs when ROS levels become too high, condition that contributes to male infertility [22]. We evaluated the sORP, a marker of oxidative stress, in SU- and DGCsperm samples and found increased values in DGCsperm compared to SU-sperm. Differences in the redox state of patients sperm processed by SU and DGC have been confirmed by a recent investigation [23]. As higher sORP values indicate higher oxidative stress, it seems DGC may induce more oxidative stress to sperm than SU. However, a previous investigation suggested DGC removes ROS and selects motile spermatozoa without enhancing oxidative stress [24]. Interestingly, despite the higher sORP values observed, DGC-sperm exhibited adequate values of sperm function and a higher degree of capacitation compared to SU-sperm right after processing. There is an overall agreement that ROS are essential for tyrosine phosphorylation of sperm proteins during capacitation [25,26], so the higher sORP levels in DGC-sperm is consistent with the higher degree of capacitation observed in these samples. Physiological levels of ROS for processed sperm maintained in a defined medium have not been characterized, so the sORP values here described may be adequate under in vitro capacitation conditions, but the biological relevance of sORP differences between SU- and DGC-sperm and its potential impact on sperm functionality must await further investigations.

A new finding of this study was the comparison of the intracellular calcium oscillation patterns between SU- and DGC-sperm samples. Intracellular calcium concentration rise initiates a number of signal trans- 
duction pathways involved in motility changes and acrosome reaction as a result of sperm capacitation [27]. Calcium oscillations are versatile signals that regulate diverse cellular processes. Specific information produced by calcium oscillations through their frequency, amplitude and kinetics are decoded by cellular molecular detectors that change their activities accordingly. Interestingly, we found that DGC-sperm showed calcium oscillations of higher frequency and amplitude than SU-sperm, suggesting that different signalling pathways may be triggered inside the cells.

In contrast, both sperm processing techniques were equally efficient in recovering motile spermatozoa, but DGC-sperm exhibited more hyperactivation and tyrosine phosphorylation than SU-sperm. Moreover, these changes were accompanied by a higher responsiveness to calcium ionophore induction of acrosome reaction, all of which indicate DGC-sperm are more capacitated than SU-sperm. This observation is in agreement with previous investigations of our group indicating that DGC removes proteins associated with the spermatozoa plasma membrane more efficiently than SU [15]. The fact that we found no significant differences in protein tyrosine phosphorylation at 6 hours suggests that differences between SU and DGC in this parameter are likely to disappear at longer incubation times, although this hypothesis should be examined in the future. Hyperactivation and protein tyrosine phosphorylation rises are the best characterized features of sperm capacitation, so advances in the understanding of in vitro capacitation after SU and DGC may help to improve the efficacy and success in ART. Indeed, it has been shown that bovine intracytoplasmic sperm injections using spermatozoa recovered by DGC and pre-incubated under capacitating conditions promote pronucleus formation rate and blastocyst rate [28].

The current study has some limitations. First, some of the differences observed between SU- and DGCsperm disappeared at 6 hours incubations and therefore may not be relevant when sperm are meant to be used few hours after processing; consequently, the processing method and optimal moment when processed sperm must be employed should be determined according to the procedure the sample will be used for. Another limitation is that the effects of SU and DGC were evaluated on normozoospermic semen samples only and consequently it is not possible to foreseen their effects on semen samples from patients with pa- thologies of different aetiology. Besides, other sperm function variables not included in this study may also be relevant to fully evaluate sperm processed by SU and DGC.

\section{CONCLUSIONS}

Results herein presented indicate the presence of functional and capacitation differences between spermatozoa recovered by SU and DGC. Furthermore, DGCsperm seem to be more capacitated than SU-sperm and therefore might be more suitable for intracytoplasmic sperm injection, while SU-sperm should be preferred for techniques such as intrauterine insemination and in vitro fertilization, where sperm full capacitation may be accomplished during female tract residency and coincubation with the cumulus-oocyte complex, respectively. In any case, differences between sperm samples retrieved by these two methods should be considered for a better selection of the semen processing method, whether for research or clinical purposes.

\section{ACKNOWLEDGEMENTS}

This study was partially supported by funds from Departamento de Biología de la Reproducción Dr. Carlos Gual Castro (Instituto Nacional de Ciencias Médicas y Nutrición Salvador Zubirán, Ciudad de México, Mexico) to MC and FL, and PAPIIT-DGAPA-UNAM (No. 202519) to CT.

The authors wish to acknowledge M. Sc. Yadira Libertad Hernández-Rueda and B. Sc. Israel Jiménez for their expert technical assistance. We also thank Dr. Pedro Caballero-Campo for his critical review of the manuscript.

\section{Conflict of interest}

The authors have nothing to disclose.

\section{Authors Contributions}

Conceptualization: MC. Data curation: GHS, ASLT. Formal analysis: GHS, ASLT, FL, VTF, CLT, MC. Funding acquisition: MC, FL, CLT. Investigation: GHS, ASLT, IMR, EMM. Methodology: GHS, ASLT, IMR, EMM. Supervision: MC. Writing - original draft: MC. Writing - review \& editing: MC, GHS, ASLT, FL, CLT, VTF. 


\section{Supplementary Materials}

Supplementary materials can be found via https://doi. org/10.5534/wjmh.200115.

\section{Data Sharing Statement}

The data required to reproduce these findings cannot be shared at this time as the data also forms part of an ongoing study.

\section{REFERENCES}

1. World Health Organization. WHO laboratory manual for the examination and processing of human semen. 5th ed. Geneva: World Health Organization; 2010.

2. Allamaneni SS, Agarwal A, Rama S, Ranganathan P, Sharma RK. Comparative study on density gradients and swim-up preparation techniques utilizing neat and cryopreserved spermatozoa. Asian J Androl 2005;7:86-92.

3. Monqaut AL, Zavaleta C, López G, Lafuente R, Brassesco M. Use of high-magnification microscopy for the assessment of sperm recovered after two different sperm processing methods. Fertil Steril 2011;95:277-80.

4. Yamanaka M, Tomita K, Hashimoto S, Matsumoto H, Satoh $\mathrm{M}$, Kato $\mathrm{H}$, et al. Combination of density gradient centrifugation and swim-up methods effectively decreases morphologically abnormal sperms. J Reprod Dev 2016;62:599-606.

5. Tachawiwat K, Getpook C, Geater A. Comparison of hyaluronan binding assay scores of spermatozoa using swim-up techniques and density gradient centrifugation. J Med Assoc Thai 2015;98 Suppl 2:S84-91.

6. Zhao F, Yang Q, Shi S, Luo X, Sun Y. Semen preparation methods and sperm telomere length: density gradient centrifugation versus the swim up procedure. Sci Rep 2016;6:39051.

7. Ricci G, Perticarari S, Boscolo R, Montico M, Guaschino S, Presani G. Semen preparation methods and sperm apoptosis: swim-up versus gradient-density centrifugation technique. Fertil Steril 2009;91:632-8.

8. Jayaraman V, Upadhya D, Narayan PK, Adiga SK. Sperm processing by swim-up and density gradient is effective in elimination of sperm with DNA damage. J Assist Reprod Genet 2012;29:557-63.

9. Puga Molina LC, Luque GM, Balestrini PA, Marín-Briggiler CI, Romarowski A, Buffone MG. Molecular basis of human sperm capacitation. Front Cell Dev Biol 2018;6:72.

10. López-Torres AS, Chirinos M. Modulation of human sperm capacitation by progesterone, estradiol, and luteinizing hor- mone. Reprod Sci 2017;24:193-201.

11. Hernández-Silva G, Chirinos M. Proteins from male and female reproductive tracts involved in sperm function regulation. Zygote 2019;27:5-16.

12. Ghaleno LR, Valojerdi MR, Janzamin E, Chehrazi M, Sharbatoghli M, Yazdi RS. Evaluation of conventional semen parameters, intracellular reactive oxygen species, DNA fragmentation and dysfunction of mitochondrial membrane potential after semen preparation techniques: a flow cytometric study. Arch Gynecol Obstet 2014;289:173-80.

13. Muratori M, Tarozzi N, Carpentiero F, Danti S, Perrone FM, Cambi M, et al. Sperm selection with density gradient centrifugation and swim up: effect on DNA fragmentation in viable spermatozoa. Sci Rep 2019;9:7492.

14. López-Torres AS, González-González ME, Mata-Martínez E, Larrea F, Treviño CL, Chirinos M. Luteinizing hormone modulates intracellular calcium, protein tyrosine phosphorylation and motility during human sperm capacitation. Biochem Biophys Res Commun 2017;483:834-9.

15. Hernández-Silva G, Fabián López-Araiza JE, López-Torres AS, Larrea F, Torres-Flores V, Chirinos M. Proteomic characterization of human sperm plasma membrane-associated proteins and their role in capacitation. Andrology 2020;8:17180

16. Agarwal A, Sharma R, Roychoudhury S, Du Plessis S, Sabanegh E. MiOXSYS: a novel method of measuring oxidation reduction potential in semen and seminal plasma. Fertil Steril 2016;106:566-73.e10.

17. Mata-Martínez E, José O, Torres-Rodríguez P, Solís-López A, Sánchez-Tusie AA, Sánchez-Guevara Y, et al. Measuring intracellular $\mathrm{Ca} 2+$ changes in human sperm using four techniques: conventional fluorometry, stopped flow fluorometry, flow cytometry and single cell imaging. J Vis Exp 2013;(75):e50344.

18. Caballero-Campo P, Chirinos M, Fan XJ, González-González ME, Galicia-Chavarría M, Larrea F, et al. Biological effects of recombinant human zona pellucida proteins on sperm function. Biol Reprod 2006;74:760-8.

19. Björndahl L, Mohammadieh M, Pourian M, Söderlund I, Kvist U. Contamination by seminal plasma factors during sperm selection. J Androl 2005;26:170-3.

20. Luppi S, Martinelli M, Giacomini E, Giolo E, Zito G, Garcia $\mathrm{RC}$, et al. Comparative proteomic analysis of spermatozoa isolated by swim-up or density gradient centrifugation. Reprod Biol Endocrinol 2015;13:36.

21. Leclerc P, de Lamirande E, Gagnon C. Regulation of proteintyrosine phosphorylation and human sperm capacitation by reactive oxygen derivatives. Free Radic Biol Med 1997;22:643- 
56.

22. Agarwal A, Sharma RK, Nallella KP, Thomas AJ Jr, Alvarez JG, Sikka SC. Reactive oxygen species as an independent marker of male factor infertility. Fertil Steril 2006;86:878-85.

23. Gode F, Gürbüz AS, Tamer B, Pala I, Isik AZ. The effects of microfluidic sperm sorting, density gradient and swimup methods on semen oxidation reduction potential. Urol J 2020;17:397-401.

24. Takeshima T, Yumura Y, Kuroda S, Kawahara T, Uemura H, Iwasaki A. Effect of density gradient centrifugation on reactive oxygen species in human semen. Syst Biol Reprod Med 2017;63:192-8.

25. Aitken RJ, Paterson M, Fisher H, Buckingham DW, van Duin
M. Redox regulation of tyrosine phosphorylation in human spermatozoa and its role in the control of human sperm function. J Cell Sci 1995;108(Pt 5):2017-25.

26. Du Plessis SS, Agarwal A, Halabi J, Tvrda E. Contemporary evidence on the physiological role of reactive oxygen species in human sperm function. J Assist Reprod Genet 2015;32:509-20.

27. Breitbart H. Intracellular calcium regulation in sperm capacitation and acrosomal reaction. Mol Cell Endocrinol 2002;187:139-44

28. Águila L, Zambrano F, Arias ME, Felmer R. Sperm capacitation pretreatment positively impacts bovine intracytoplasmic sperm injection. Mol Reprod Dev 2017;84:649-59. 